\title{
Review of: "Is it of any positive being COVID-19 positive?: Cross-protection hypothesis"
}

\section{Mohammed Jaber ${ }^{1}$}

1 Cairo University

Potential competing interests: The author(s) declared that no potential competing interests exist.

Thank you for sharing 